\title{
Multitarget Tracking by Improved Particle Filter Based on $H_{\infty}$ Unscented Transform
}

\author{
Yazhao Wang \\ The Department of Systems and Control, Beihang University (BUAA), Beijing 100191, China \\ Correspondence should be addressed to Yazhao Wang; bhbwc2012@126.com
}

Received 29 August 2013; Accepted 11 October 2013

Academic Editor: Tao Li

Copyright (C) 2013 Yazhao Wang. This is an open access article distributed under the Creative Commons Attribution License, which permits unrestricted use, distribution, and reproduction in any medium, provided the original work is properly cited.

\begin{abstract}
This paper considers the problem of multitarget tracking in cluttered environment. To reduce the dependency on the noise priori knowledge, an improved particle filtering (PF) data association approach is presented based on the $H_{\infty}$ filter (HF). This approach can achieve higher robustness in the condition that the measurement noise prior is unknown. Because of the limitations of the HF in nonlinear tracking, we first present the $H_{\infty}$ unscented filter (HUF) by embedding the unscented transform (UT) into the $H_{\infty}$ extended filter (HEF) structure. Then the HUF is incorporated into the Rao-Blackwellized particle filter (RBPF) framework to update the particles. Simulation results are provided to demonstrate the effectiveness of the proposed algorithms in linear and nonlinear multitarget tracking.
\end{abstract}

\section{Introduction}

Multitarget tracking is to estimate the targets' current positions from a series of noise-corrupted measurements by filtering methods [1]. In cluttered environment, the foremost difficulty involves the problem of associating the correct measurements with the appropriate tracks. A number of strategies, including the joint probabilistic data association (JPDA) [2,3], multiple hypothesis tracking (MHT) [4, 5], S-D assignment [6], and the probabilistic hypothesis density filer (PHDF) [7-9], are available to solve this problem.

Recently, the sequential Monte Carlo (SMC) data association approaches are also applied to the tracking and association problems $[10,11]$. This paper tackles data association joint with state estimation via a SMC method called RaoBlackwellized particle filter (RBPF) [12-14]. This particle method can be considered as a generalization of MHT, which represents the data association and state posteriors as a discrete set of hypotheses. Instead of the pure particle strategy, a mixture of Gaussian representation of the joint posterior distribution is used to reduce the estimation variance. In the application of RBPF for nonlinear target tracking, it can replace the Kalman filter (KF) in the data association algorithm by the extended Kalman filter (EKF) or the unscented Kalman filter (UKF) [15]. Although RBPF has shown higher tracking efficiency against the pure particle filtering schemes, there is still weakness lying in the strong assumptions on the system models and noise statistics [16]. That is, the KF or its variants can only be applied in the condition that a perfect system model is known and the process and measurement noise statistics are white and Gaussian with known covariance matrices. In many practical applications, these assumptions can hardly be satisfied, and the performance of the KF based methods may degrade severely when the measurement disturbances are not in definite Gaussian forms. Hence, we need a solution with more robustness against the uncertainties of the noise than the KF. The $H_{\infty}$ filter (HF), which aims at minimizing the worst possible effects of the disturbances on the estimation errors, can provide an alternative to solve this problem [1719]. Different from the KF, the only assumption made for HF is that the noise signals have a finite energy but without any statistic assumptions.

The HF techniques have been used in linear-model target tracking. Accordingly, the $H_{\infty}$ extended filter (HEF) has also been proposed for nonlinear models by using the EKF structure. As another way to approximate the filtering distribution, the unscented transform (UT) can be more 
accurate compared to the EKF for it performs a higher order of the Taylor series expansion. By a Gaussian density instead of approximating the nonlinear functions as the EKF, the UT technique has shown its priority in handling nonlinear estimation problems and also been combined with the $H_{\infty}$ filter [20]. In this paper, we aim to incorporate the HF technique into the framework of RBPF to reduce its dependency on a priori knowledge of the noise statistics in multitarget tracking. In RBPF, the continuous state is estimated using the KF or its variants, while the discrete state or the mode state is estimated using particle filters. Thus, the HF can be embedded into the RBPF structure directly. Because of the limitations of the HF in nonlinear tracking, we present the $H_{\infty}$ unscented filter (HUF) by embedding the UT into the HEF structure. Since the HUF has the same observer structure as the UKF, it can also be embedded into the RBPF framework to update the continuous states of the particles.

The remainder of this paper is organized as follows. In Section 2, we give a brief introduction of the generic RBPF target tracking approach and the basic $H_{\infty}$ linear filter structure. The main work is given in Section 3 where the HUF is first presented. Then the HUF is incorporated into the RBPF framework to update the particles. Simulation results are provided in Section 4 to demonstrate the effectiveness of the proposed algorithms in linear and nonlinear multitarget tracking. In Section 5, conclusions are also given to summarize the main works of this paper.

\section{Background}

2.1. Rao-Blackwellized Particle Filter for Target Tracking. Consider the following time-varying state-space system:

$$
\begin{gathered}
\mathbf{x}_{k}=\mathbf{F}_{k-1} \mathbf{x}_{k-1}+\mathbf{w}_{k-1}, \\
\mathbf{z}_{k}=\mathbf{H}_{k} \mathbf{x}_{k}+\mathbf{v}_{k}
\end{gathered}
$$

where $\mathbf{x}_{k} \in \mathbb{R}^{n}$ and $\mathbf{z}_{k} \in \mathbb{R}^{p}$ are the system state and measurement vectors at time step $k$, respectively. $\mathbf{w}_{k-1} \sim$ $\mathcal{N}\left(0, \mathbf{Q}_{k-1}\right)$ and $\mathbf{v}_{k} \sim \mathcal{N}\left(0, \mathbf{R}_{k}\right)$ are zero mean mutually independent Gaussian process noise, and $\mathbf{F}_{k-1}$ and $\mathbf{H}_{k}$ are matrices with compatible dimensions. Suppose that we are able to form another variable $c_{k}$ to describe the matrices $\mathbf{F}_{k-1}$ and $\mathbf{H}_{k}$; then the RBPF algorithm can be applied to estimate the whole state $\left\{\mathbf{x}_{k} ; c_{k}\right\}$ [21]. For space consideration, we omit the details of the RBPF algorithms which can be found in $[22,23]$. In the application of RBPF for target tracking, the latent variable $c_{k}$ is defined to be the data association event indicator [12]. That is, $c_{k}=0$ when the measurement is from clutter, and $c_{k}=j$ when the measurement is from target $j$ ( $j=1, \ldots, T$, where $T$ is number of targets). The predictive probability $p\left(c_{k} \mid c_{1: k-1}\right)$ gives the data association prior given the data association results $\left\{c_{1: k-1}\right\}$ in the previous $k-1$ time steps. The posterior distribution of $c_{k}$ is $p\left(c_{k} \mid c_{1: k-1}, \mathbf{z}_{1: k}\right)$ which can be calculated by

$$
\begin{aligned}
& p\left(c_{k} \mid c_{1: k-1}, \mathbf{z}_{1: k}\right) \\
& \quad \propto p\left(\mathbf{z}_{k} \mid c_{k}, c_{1: k-1}, \mathbf{z}_{1: k-1}\right) p\left(c_{k} \mid c_{1: k-1}\right),
\end{aligned}
$$

where the data association prior $p\left(c_{k} \mid c_{1: k-1}\right)$ is modeled as a recursive Markov chain, which guarantees the association assumption of one target per measurement in each time step. Accordingly, the $p\left(c_{k} \mid c_{1: k-1}\right)$ in (2) has been replaced by $p\left(c_{k} \mid c_{k-M: k-1}\right)$ [13], and the general form of the joint prior model is given by

$$
p\left(c_{k+M-1}, \ldots, c_{k}\right)=\prod_{m=1}^{M} p\left(c_{k+m} \mid c_{k}, \ldots, c_{k+m-1}\right) .
$$

That means if we obtain $M$ measurements in time step $k$, the $m$ th $(m=2, \ldots, M)$ measurement's association prior $p\left(c_{k+m-1} \mid c_{k+m-2}, \ldots, c_{k}\right)$ only depends on the previous association results $\left\{c_{k+m-2}, \ldots, c_{k}\right\}$ in the $k$ th time step. Note that $c_{k}$ has the prior $p\left(c_{k}\right)$ if $m=1$. This RBPF multitarget tracking algorithm is also termed Rao-Blackwellized Monte Carlo data association (RBMCDA) [12]. In this paper, we aim to improve the robustness of the RBPF for target tracking by the $H_{\infty}$ filter and unscented transform.

2.2. $H_{\infty}$ Linear Filter. Consider the model given by (1), where the process noise $\mathbf{w}_{k-1}$ and the measurement noise $\mathbf{v}_{k}$ are assumed to be uncorrelated zero-mean white noise processes with unknown statistical properties. Note that they are also the energy bounded $l_{2}[0,+\infty]$ signals. Let $\widehat{\mathbf{x}}_{k \mid k} \triangleq \mathscr{L}\left\{\mathbf{z}_{0}, \mathbf{z}_{1}, \ldots, \mathbf{z}_{k}\right\}$ denote the estimation of $\mathbf{x}_{k}$ given measurements $\mathbf{z}_{0: k}$. We can define the estimation error as $\mathscr{E}_{k} \triangleq \widehat{\mathbf{x}}_{k \mid k}-\mathbf{x}_{k}$ and denote $\mathscr{T}_{k}(\mathscr{L})$ as the transfer operator that maps the unknown disturbances $\mathbf{x}_{0}-\widehat{\mathbf{x}}_{0 \mid 0}, \mathbf{w}_{0: k-1}$, and $\mathbf{v}_{1: k}$ to the estimation errors $\mathscr{E}_{1: k}$, where $\widehat{\mathbf{x}}_{0 \mid 0}$ is a priori estimate of $\mathbf{x}_{0}$ and $\mathbf{x}_{0}-\widehat{\mathbf{x}}_{0 \mid 0}$ represents unknown initial estimation error.

In optimal $H_{\infty}$ filter, it is operated to minimize the possible worst effects of the unknown disturbances on the estimation errors. That is, the estimation strategy $\mathscr{L}$ should be designed so as to minimize the $H_{\infty}$ norm of the operator $\mathscr{T}_{k}(\mathscr{L})$. Actually, it is hard to obtain the closed-form solution of the optimal $H_{\infty}$ filtering except in some specific cases. For example, the desired accuracy of the optimal $H_{\infty}$ filter can be obtained by iterating the $\gamma^{2}$ of the suboptimal solution [16]. This paper considers the suboptimal solutions that can bound the maximum energy gain from the disturbance to the estimation errors under the prescribed disturbance tolerance level. Given a scalar $\gamma^{2}>0$, find the estimation strategies $\mathscr{L}$ such that the $H_{\infty}$ norm of $\mathscr{T}_{k}(\mathscr{L})$ satisfies $[24,25]$

$$
\begin{aligned}
& \left\|\mathscr{T}_{k}(\mathscr{L})\right\|_{\infty} \\
& \triangleq \frac{\sup _{\mathbf{x}_{0}, \mathbf{w}_{t}, \mathbf{v}_{t} \in l_{2}}\left(\sum_{t=1}^{k}\left\|\mathscr{E}_{t}\right\|_{2}^{2}\right)}{\left(\left\|\mathbf{x}_{0}-\widehat{\mathbf{x}}_{0 \mid 0}\right\|_{\mathbf{P}_{0 \mid 0}^{-1}}^{2}+\sum_{j=0}^{k}\left\|\mathbf{w}_{t}\right\|_{\mathbf{Q}_{t}^{-1}}^{2}+\sum_{t=1}^{k}\left\|\mathbf{v}_{t}\right\|_{\mathbf{R}_{t}^{-1}}^{2}\right)}<\gamma^{2},
\end{aligned}
$$

where the notation $\|a\|_{\beta}^{2}$ is defined as the square of the weighted $l_{2}$ norm of $a$, that is, $\|a\|_{\beta}^{2}=a^{T} \beta a$. The matrix $\mathbf{P}_{0 \mid 0}>0$ reflects a priori knowledge of how close $\mathbf{x}_{0}$ is to the initial estimate $\widehat{\mathbf{x}}_{0 \mid 0} \cdot \mathbf{Q}_{t}>0$ and $\mathbf{R}_{t}>0$ are weighting matrices, by which the designer can make appropriate choice 
to satisfy the performance requirements. The solution to the $H_{\infty}$ filtering algorithm as shown in [26] is given by

$$
\begin{gathered}
\widehat{\mathbf{x}}_{k \mid k-1}=\mathbf{F}_{k-1} \widehat{\mathbf{x}}_{k-1 \mid k-1}, \\
\mathbf{P}_{k \mid k-1}=\mathbf{F}_{k-1} \mathbf{P}_{k-1 \mid k-1} \mathbf{F}_{k-1}^{T}+\mathbf{Q}_{k-1}, \\
\widehat{\mathbf{x}}_{k \mid k}=\widehat{\mathbf{x}}_{k \mid k-1}+\mathbf{K}_{k}\left(\mathbf{z}_{k}-\mathbf{H}_{k} \widehat{\mathbf{x}}_{k \mid k-1}\right), \\
\mathbf{K}_{k}=\mathbf{P}_{k \mid k-1} \mathbf{H}_{k}^{T}\left[\begin{array}{l}
\mathbf{R}_{k}+\mathbf{H}_{k} \mathbf{P}_{k \mid k-1} \mathbf{H}_{k}^{T}
\end{array}\right]^{-1}, \\
\mathbf{P}_{k \mid k}=\mathbf{P}_{k \mid k-1}-\mathbf{P}_{k \mid k-1}\left[\begin{array}{ll}
\mathbf{H}_{k}^{T} & I
\end{array}\right] R_{\mathscr{C}, k}^{-1}\left[\begin{array}{ll}
\mathbf{H}_{k}^{T} & I
\end{array}\right]^{T} \mathbf{P}_{k \mid k-1},
\end{gathered}
$$

where $I$ is an identity matrix with compatible dimension. The matrix $R_{\mathscr{E}, k}$ is given by

$$
R_{\mathscr{C}, k}=\left[\begin{array}{cc}
\mathbf{R}_{k} & 0 \\
0 & -\gamma^{2} I
\end{array}\right]+\left[\begin{array}{c}
\mathbf{H}_{k} \\
I
\end{array}\right] \mathbf{P}_{k \mid k-1}\left[\begin{array}{ll}
\mathbf{H}_{k}^{T} & I
\end{array}\right] .
$$

\section{Improved Rao-Blackwellized Particle Filter}

3.1. $H_{\infty}$ Nonlinear Filter with Unscented Transform. Consider the following discrete-time nonlinear state-space model:

$$
\begin{gathered}
\mathbf{x}_{k}=f\left(\mathbf{x}_{k-1}\right)+\mathbf{w}_{k-1}, \\
\mathbf{z}_{k}=h\left(\mathbf{x}_{k}\right)+\mathbf{v}_{k} .
\end{gathered}
$$

The HEF just replaces $\mathbf{F}_{k-1}$ and $\mathbf{H}_{k}$ in (5) and (7) by $f\left(\widehat{\mathbf{x}}_{k-1 \mid k-1}\right)$ and $h\left(\widehat{\mathbf{x}}_{k \mid k-1}\right)$, respectively. Here, we present the HUF by embedding the UT technique into the HF structure, which can be used to update the continuous-state particles in the RBPF framework. Suppose that $(2 n+1)$ sigma points are generated based on the state estimates at time $k-1$,

$$
\begin{gathered}
\zeta_{k-1 \mid k-1}^{0}=\widehat{\mathbf{x}}_{k-1 \mid k-1}, \\
\zeta_{k-1 \mid k-1}^{s}=\widehat{\mathbf{x}}_{k-1 \mid k-1}+\left(\sqrt{(n+\mathscr{D}) \mathbf{P}_{k-1 \mid k-1}}\right)^{s} \\
\zeta_{k-1 \mid k-1}^{s+n}=\widehat{\mathbf{x}}_{k-1 \mid k-1}-\left(\sqrt{(n+\mathscr{D}) \mathbf{P}_{k-1 \mid k-1}}\right)^{s}, \quad s=1, \ldots, n,
\end{gathered}
$$

where the state estimate $\widehat{\mathbf{x}}_{k-1 \mid k-1}$ and its covariance $\mathbf{P}_{k-1 \mid k-1}$ have been obtained at time $k-1 . \mathscr{D} \in \mathbb{R}$ is a scaling factor, and $\left(\sqrt{(n+\mathscr{D}) \mathbf{P}_{k-1 \mid k-1}}\right)^{s}$ is the $s$ th row or column of the matrix squares root of $(n+\mathscr{D}) \mathbf{P}_{k-1 \mid k-1}$. By implementing the UT into (5) and (6), the predicted mean and covariance can be obtained as follows:

$$
\begin{gathered}
\zeta_{k \mid k-1}^{s}=f\left(\zeta_{k-1 \mid k-1}^{s}\right), \\
\widehat{\mathbf{x}}_{k \mid k-1}=\sum_{s=0}^{2 n} \omega^{s} \zeta_{k \mid k-1}^{s}, \\
\mathbf{P}_{k \mid k-1}=\sum_{s=0}^{2 n} \omega^{s}\left[\zeta_{k \mid k-1}^{s}-\widehat{\mathbf{x}}_{k \mid k-1}\right] \\
\times\left[\zeta_{k \mid k-1}^{s}-\widehat{\mathbf{x}}_{k \mid k-1}\right]^{T}+\mathbf{Q}_{k-1},
\end{gathered}
$$

where $\omega^{s}$ is the normalized weight associated with the sth sigma points (see $[27,28]$ ). Since the linearized measurement function $\mathbf{H}_{k}$ does not exist explicitly under the unscented transform framework, the statistical linear error propagation method [29] is used to reformulate the updated equations. Approximately, the measurement covariance and its crosscorrelation covariance can be given by

$$
\begin{gathered}
\mathbf{P}_{k \mid k-1}^{z z} \approx \mathbf{H}_{k} \mathbf{P}_{k \mid k-1} \mathbf{H}_{k}^{T}, \\
\mathbf{P}_{k \mid k-1}^{x z} \approx \mathbf{P}_{k \mid k-1} \mathbf{H}_{k}^{T} .
\end{gathered}
$$

By using the predicted sigma points, they can be calculated by

$$
\begin{aligned}
& \mathbf{P}_{k \mid k-1}^{z z}=\sum_{s=0}^{2 n} \omega^{s} {\left[h\left(\zeta_{k \mid k-1}^{s}\right)-\widehat{\mathbf{z}}_{k \mid k-1}\right] } \\
& \times {\left[h\left(\zeta_{k \mid k-1}^{s}\right)-\widehat{\mathbf{z}}_{k \mid k-1}\right]^{T}, } \\
& \mathbf{P}_{k \mid k-1}^{x z}=\sum_{s=0}^{2 n} \omega^{s}\left[\zeta_{k \mid k-1}^{s}-\widehat{\mathbf{x}}_{k \mid k-1}\right] \\
& \times\left[h\left(\zeta_{k \mid k-1}^{s}\right)-\widehat{\mathbf{z}}_{k \mid k-1}\right]^{T},
\end{aligned}
$$

where

$$
\widehat{\mathbf{z}}_{k \mid k-1}=\sum_{s=0}^{2 n} \omega^{s} h\left(\zeta_{k \mid k-1}^{s}\right)
$$

By substituting (14) into (7)-(10), the filtered estimates can be obtained by

$$
\begin{aligned}
\widehat{\mathbf{x}}_{k \mid k}= & \widehat{\mathbf{x}}_{k \mid k-1}+\mathbf{P}_{k \mid k-1}^{x z}\left[\mathbf{R}_{k}+\mathbf{P}_{k \mid k-1}^{z z}\right]^{-1} \\
& \times\left(\mathbf{z}_{k}-\widehat{\mathbf{z}}_{k \mid k-1}\right) \\
\mathbf{P}_{k \mid k}= & \mathbf{P}_{k \mid k-1}-\left[\begin{array}{ll}
\mathbf{P}_{k \mid k-1}^{x z} & \mathbf{P}_{k \mid k-1}
\end{array}\right] \\
& \times \mathbf{R}_{\mathscr{E}, k}^{-1}\left[\begin{array}{ll}
\mathbf{P}_{k \mid k-1}^{x z} & \mathbf{P}_{k \mid k-1}
\end{array}\right]^{T}
\end{aligned}
$$

where

$$
R_{\mathscr{C}, k}=\left[\begin{array}{cc}
\mathbf{R}_{k}+\mathbf{P}_{k \mid k-1}^{z z} & {\left[\mathbf{P}_{k \mid k-1}^{x z}\right.} \\
\mathbf{P}_{k \mid k-1}^{x z} & -\gamma^{2} I+\mathbf{P}_{k \mid k-1}^{T}
\end{array}\right] .
$$

It should be pointed out that the HUF has the same observer structure as that of the UKF, and $\mathbf{Q}_{k-1}$ and $\mathbf{R}_{k}$ play the same role as the covariance matrices of the process noise and the measurement noise when using the UKF. Hence, the weighting matrices can be adjusted with no conflictions to the framework of KF. For the HUF, it not only outperforms the HEF in accuracy, but also achieves more robustness than the UKF for unknown noise statistics [16].

3.2. $H_{\infty}$ Unscented Rao-Blackwellized Particle Filter. In this section, the HUF based RBPF multiple target tracking algorithm (HURBPF) is provided. It can be found that 
the $p\left(\mathbf{x}_{k} \mid c_{i, 1: k}, \mathbf{z}_{1: k}\right)$ will not be strictly Gaussian if the system dynamic and (or) measurement function are (is) nonlinear. In this case, the HUF can be a better candidate than HEF or UKF for updating the continuous state within the particle filtering framework. The conditional distribution mentioned above can be chosen as

$$
p\left(\mathbf{x}_{k} \mid c_{i, 1: k}, \mathbf{z}_{1: k}\right)=\mathcal{N}\left(\mathbf{x}_{k} ; \widehat{\mathbf{x}}_{i, k \mid k}, \mathbf{P}_{i, k \mid k}\right)
$$

where $\widehat{\mathbf{x}}_{i, k \mid k}$ and $\mathbf{P}_{i, k \mid k}$ are the mean and covariance of $\mathbf{x}_{k}$ computed by the HUF (17). The main procedure for the HURBPF algorithm is presented as follows (see Algorithm 1). In this algorithm, the target state priors can be represented as a weighted importance sample set

$$
p\left(\mathbf{x}_{0}^{(j)}\right)=\sum_{i=1}^{N_{p}} \mathbf{w}_{i, 0} \mathcal{N}\left(\mathbf{x}_{0}^{(j)} \mid \mathbf{x}_{i, 0}^{(j)}, \mathbf{P}_{i, 0}^{(j)}\right)
$$

where $i\left(i=1, \ldots, N_{p}\right)$ is the identifier of particle.

Algorithm 1. $H_{\infty}$ unscented Rao-Blackwellized particle filter.

For $k=1$ to $n$ do.

Step 1. Do prediction step. For $i=1,2, \ldots, N_{p}$ and $j=$ $1,2, \ldots, T$, perform HUF prediction from the mean $\widehat{\mathbf{x}}_{i, k-1 \mid k-1}$ and covariances $\mathbf{P}_{i, k-1 \mid k-1}$ to generate the predicted estimates $\widehat{\mathbf{x}}_{i, k \mid k-1}^{(j)}$ and $\mathbf{P}_{i, k \mid k-1}^{(j)}$.

Step 2. Calculate the data association priors $p\left(c_{i, k} \mid c_{i, 1: k-1}\right)$ based on the recursive Markov chain described in [13].

Step 3. For $i=1,2, \ldots, N_{p}$, perform HUF update for each particle to get contemporary filtered estimates $\overline{\mathbf{x}}_{i, k \mid k}^{(j)}$ and $\overline{\mathbf{P}}_{i, k \mid k}^{(j)}$.

Step 4. Calculate the posterior distribution of $c_{i, k}$ :

$$
\begin{aligned}
& p\left(c_{i, k} \mid c_{i, 1: k-1}, \mathbf{z}_{1: k}\right) \\
& \quad=p\left(\mathbf{z}_{k} \mid c_{i, k}, c_{i, 1: k-1}, \mathbf{z}_{1: k-1}\right) p\left(c_{i, k} \mid c_{i, 1: k-1}\right) .
\end{aligned}
$$

Step 5. Sample a new association $c_{i, k}=j$ with probability $\rho_{i}^{(j)}$ :

$$
\rho_{i}^{(j)}=\frac{\widehat{\rho}_{i}^{(j)}}{\sum_{j^{\prime}=0}^{T} \widehat{\rho}_{i}^{\left(j^{\prime}\right)}}, \quad j=0, \ldots, T,
$$

where

$$
\begin{aligned}
& \hat{\rho}_{i}^{(0)}=p\left(c_{i, k}=0 \mid c_{i, 1: k-1}, \mathbf{z}_{1: k}\right), \\
& \hat{\rho}_{i}^{(j)}=p\left(c_{i, k}=j \mid c_{i, 1: k-1}, \mathbf{z}_{1: k}\right) .
\end{aligned}
$$

Step 6. Calculate the new weights

$$
\mathbf{w}_{i, k} \propto \mathbf{w}_{i, k-1} \frac{p\left(\mathbf{z}_{k} \mid c_{i, k}, c_{i, 1: k-1}, \mathbf{z}_{1: k-1}\right) p\left(c_{i, k} \mid c_{i, 1: k-1}\right)}{p\left(c_{i, k} \mid c_{i, 1: k-1}, \mathbf{z}_{1: k}\right)} .
$$

Step 7. Update the $j$ th target according to the new $c_{i, k}(i=$ $\left.1,2, \ldots, N_{p}\right)$. If $c_{i, k}=j(j \neq 0)$, then

$$
\widehat{\mathbf{x}}_{i, k \mid k}^{(j)}=\overline{\mathbf{x}}_{i, k \mid k}^{(j)} \quad \mathbf{P}_{i, k \mid k}^{(j)}=\overline{\mathbf{P}}_{i, k \mid k}^{(j)}
$$

Step 8. Calculate the target state vectors

$$
\widehat{\mathbf{x}}_{k \mid k}^{(j)}=\sum_{i=1}^{N_{p}} \mathbf{w}_{i, k} \widehat{\mathbf{x}}_{i, k \mid k}^{(j)}
$$

End for. (Resample if needed [30-32].)

It should be pointed out that in $H_{\infty}$ filter the level $\gamma$ must be selected carefully to guarantee the existence of the HUF, or else the filtering program will fail in the applications of HURBPF approach. To adaptively adjust $\gamma$ to its minimum at each iteration, we can choose the value of $\gamma_{k}$ as [16]

$$
\begin{aligned}
\gamma_{k}^{2}=\alpha \max \{\operatorname{eig}( & \mathbf{P}_{k \mid k-1}^{-1}+\mathbf{P}_{k \mid k-1}^{-1} \mathbf{P}_{k \mid k-1}^{x z} \mathbf{R}_{\mathbf{k}}^{-1} \\
& \left.\left.\times\left[\mathbf{P}_{k \mid k-1}^{-1} \mathbf{P}_{k \mid k-1}^{x z}\right]^{T}\right)^{-1}\right\}
\end{aligned}
$$

where $\alpha$ is a scalar larger than one and $\max \left\{\operatorname{eig}(A)^{-1}\right\}$ denotes the maximum eigenvalue of the matrix $A^{-1}$.

\section{Simulation Results}

This section presents the two-dimensional (2D) target tracking examples to demonstrate the performance of the proposed tracking algorithms.

Example 1. The targets are modeled with near constant velocity model in Cartesian coordinates. The discrete-time dynamic and measurement models of the $j$ th target have the following form:

$$
\begin{gathered}
\mathbf{x}_{j, k}=\mathbf{F}_{j, k-1} \mathbf{x}_{j, k-1}+\mathbf{w}_{k-1}, \\
\mathbf{z}_{j, k}=\mathbf{H}_{j, k} \mathbf{x}_{j, k}+\mathbf{v}_{k},
\end{gathered}
$$

where

$$
\begin{aligned}
\mathbf{F}_{j, k-1} & =\left(\begin{array}{cccc}
1 & 0 & \delta_{t} & 0 \\
0 & 1 & 0 & \delta_{t} \\
0 & 0 & 1 & 0 \\
0 & 0 & 0 & 1
\end{array}\right), \\
\mathbf{H}_{j, k} & =\left(\begin{array}{llll}
1 & 0 & 0 & 0 \\
0 & 1 & 0 & 0
\end{array}\right),
\end{aligned}
$$

$\mathbf{w}_{k-1}$ and $\mathbf{v}_{k}$ are zero mean Gaussian process noises. The standard process and measurement noise variances are selected as $\sigma_{x}=0.001 \mathrm{~km}$ and $\sigma_{z}=20 \mathrm{~km}$, respectively. The sample interval $\delta_{t}=1$ and the correct measurements return with a known detection probability $P_{d}=$ 1 (detection missing will not happen). At each time step $k$, the target is located at coordinates $\left(x_{k}, y_{k}\right)$ and moves with constant velocity vector $\left(\dot{x}_{k}, \dot{y}_{k}\right)$, which are combined 


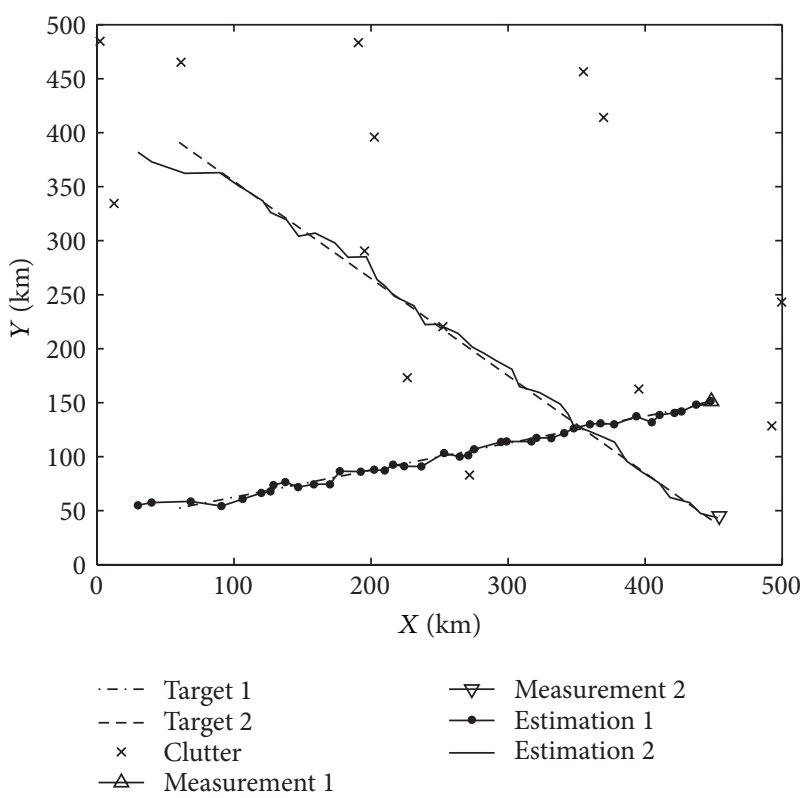

FIGURE 1: Example of two targets tracking (a successful track by HRBPF).

with the target's state vector $\mathbf{x}_{k}=\left(x_{k}, y_{k}, \dot{x}_{k}, \dot{y}_{k}\right)^{T}$. In this two-target-crossing scenario (see Figure 1), target 1 and target 2 begin at $k=0$ with position-velocity coordinates $\mathbf{x}_{0}^{(1)}=(50 \mathrm{~km}, 50 \mathrm{~km}, 10 \mathrm{~km} / \mathrm{s}, 2.5 \mathrm{~km} / \mathrm{s})^{T}$ and $\mathbf{x}_{0}^{(2)}=$ $(50 \mathrm{~km}, 400 \mathrm{~km}, 10 \mathrm{~km} / \mathrm{s},-9 \mathrm{~km} / \mathrm{s})^{T}$, respectively. The total number of tracking time steps is 40 .

The KRBPF serves as the baseline algorithm, and the proposed HRBPF algorithm is compared with it. Both algorithms are designed based on the same assumptions, and the performance of the two algorithms is evaluated by the average results over Monte Carlo runs. The initial state estimates of the two targets are set to $\widehat{\mathbf{x}}_{0 \mid 0}^{(1)}=(30 \mathrm{~km}, 50 \mathrm{~km}, 10 \mathrm{~km} / \mathrm{s}, 2.5 \mathrm{~km} / \mathrm{s})^{T}$ and $\widehat{\mathbf{x}}_{0 \mid 0}^{(2)}=$ $(30 \mathrm{~km}, 400 \mathrm{~km}, 10 \mathrm{~km} / \mathrm{s},-9 \mathrm{~km} / \mathrm{s})^{T}$, respectively. The clutter is modeled as independent and identically distributed with uniform spatial distribution in a detection region of the coordinate plane $[0 \mathrm{~km}, 500 \mathrm{~km}] \times[0 \mathrm{~km}, 500 \mathrm{~km}]$, and the number of clutter measurements obeys a Poisson distribution with the Poisson random number $\lambda=10$ (clutter rate). The number of particles used in the simulation is 100 . The standard measurement variance used in both algorithms, KRBPF (Kalman filter based RBPF) and HRBPF $\left(H_{\infty}\right.$ filter based RBPF), is $\sigma_{z}^{a}=30 \mathrm{~km}$. The position root mean square errors (RMSEs) of the KRBPF and HRBPF algorithms are illustrated in Figure 2 (target 1) and Figure 3 (target 2). We can see that the overall performance of the proposed HRBPF is significantly better than that of the KRBPF as expected. This is due to the fact that the HF outperforms the KF when the statistics of the noise processes are not known by the filtering algorithms.

Example 2. Consider a scenario of tracking two targets using bearings-only measurements received by two static sensors

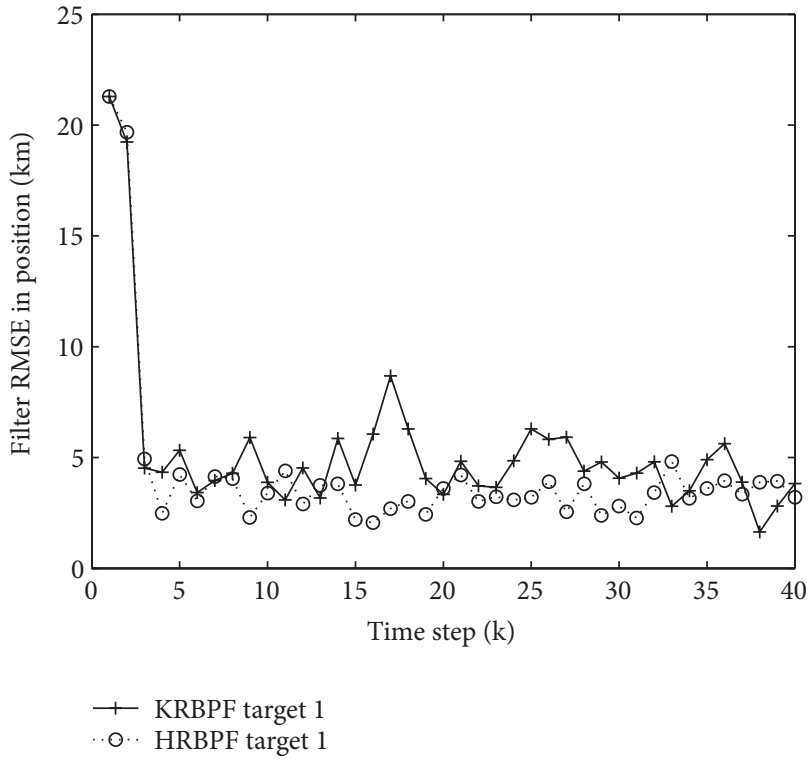

FIGURE 2: Comparison of the average position estimation errors of target 1 .

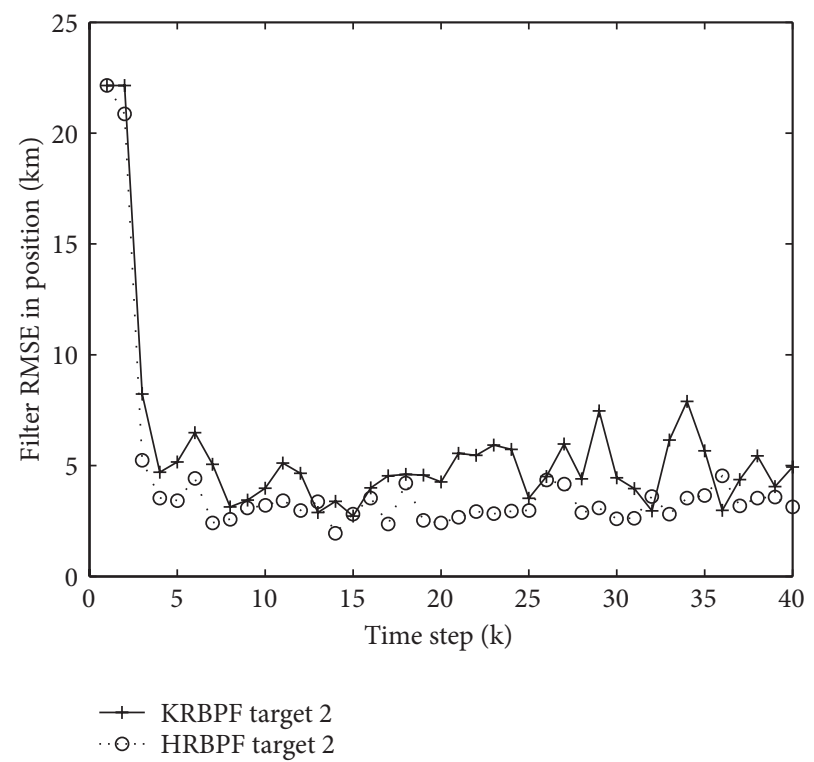

FIgURE 3: Comparison of the average position estimation errors of target 2 .

which are located at $\left(s_{x, k}^{i}, s_{y, k}^{i}\right), i=1,2$ (see Figure 4 , where “ $\square$ " represents the location of the sensor). The dynamic of discrete-time velocity model is the same as (28), and the measurement function is given by

$$
z_{j, k}^{i}=\arctan \left(\frac{y_{j, k}-s_{y, k}^{i}}{x_{j, k}-s_{x, k}^{i}}\right)+r_{k}^{i},
$$

where $r_{k}^{i} \sim N\left(0, \sigma_{z}^{2}\right)$ with $\sigma_{z}=0.01 \mathrm{rad}$. Since this measurement model is nonlinear, the URBPF (UKF based RBPF) and HURBPF are employed for tracking. The clutter originated 


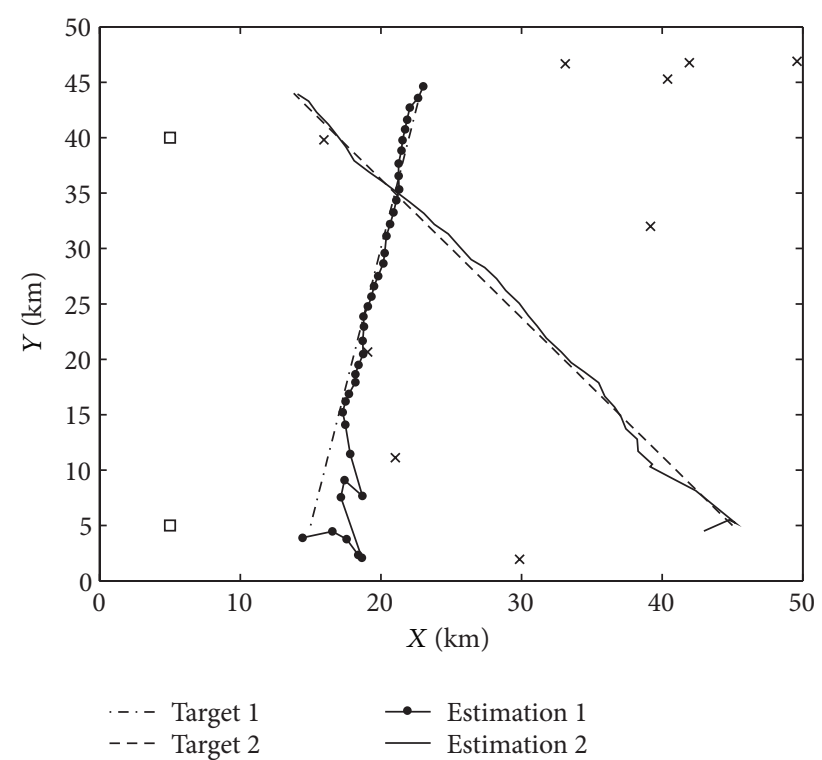

FIGURE 4: Example of two targets bearings-only tracking (a successful track by HURBPF).

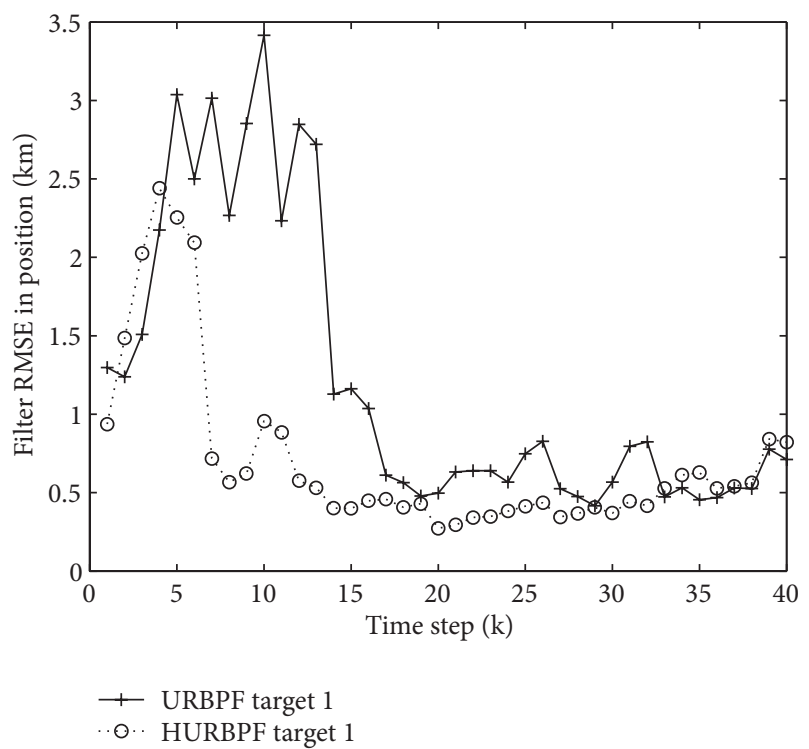

FIGURE 5: Comparison of the average position estimation errors of target 1.

measurements obey Poisson distribution with clutter rate $\lambda=$ 5 in a single time step. The detection region of the coordinated plane is set to be $[0 \mathrm{~km}, 50 \mathrm{~km}] \times[0 \mathrm{~km}, 50 \mathrm{~km}]$. The standard bearings-only measurement variance used in both algorithms is $\sigma_{z}^{a}=0.03 \mathrm{rad}$.

The performance of the HURBPF is compared with that of the URBPF using 50 particles. The two targets begin at $k=0$ with position-velocity coordinates $\mathbf{x}_{0}^{(1)}=$ $(15 \mathrm{~km}, 5 \mathrm{~km}, 0.2 \mathrm{~km} / \mathrm{s}, 1 \mathrm{~km} / \mathrm{s})^{T}$ and $\mathbf{x}_{0}^{(2)}=(45 \mathrm{~km}, 5 \mathrm{~km}$, $-0.8 \mathrm{~km} / \mathrm{s}, 1 \mathrm{~km} / \mathrm{s})^{T}$, respectively. The total number of tracking time steps is 40 . In this example, the initial state

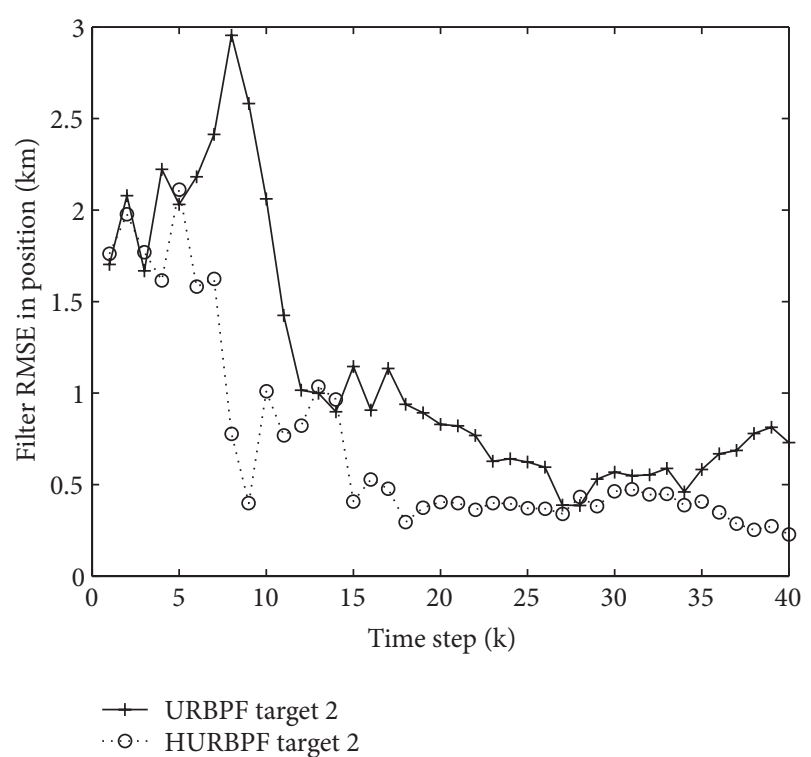

FIgURE 6: Comparison of the average position estimation errors of target 2 .

estimates of the two targets are set to $\widehat{\mathbf{x}}_{0 \mid 0}^{(1)}=(13 \mathrm{~km}, 3 \mathrm{~km}$, $0.05 \mathrm{~km} / \mathrm{s}, 0 \mathrm{~km} / \mathrm{s})^{T}$ and $\widehat{\mathbf{x}}_{0 \mid 0}^{(2)}=(43 \mathrm{~km}, 3 \mathrm{~km},-0.2 \mathrm{~km} / \mathrm{s}$, $0 \mathrm{~km} / \mathrm{s})^{T}$.

The tracking performance of the HURBPF and the URBPF in terms of RMSE in position is shown in Figures 5 and 6 . It can be seen that the HURBPF also outperforms the URBPF for almost the entire simulation interval since the HURBPF deals with the unknown measurement noises variance priors. By the typical examples demonstrated above, a conclusion can be drawn that the $H_{\infty}$ based RBPF algorithms show good robustness against unknown noise statistics.

\section{Conclusions}

In this paper, we present an improved Rao-Blackwellized particle filtering algorithm by using the $H_{\infty}$ unscented transform. The main benefit lies in it requiring no priori knowledge of the statistical properties of the measurement noise. By decomposing the RBPF filtering distribution, the HRBPF and HURBPF algorithms are developed based on the $H_{\infty}$ filter for solving the multitarget tracking problems with unknown noise statistics. The proposed algorithms are tested by both the linear and nonlinear tracking experiments. Simulation results show that they can achieve better tracking performance than the standard KF and UKF based RBPF algorithms.

\section{Acknowledgment}

The author would like to thank the anonymous reviewers for their helpful comments on this paper. 


\section{References}

[1] M. Mallick, B. N. Vo, T. Kirubarajan, and S. Arulampalam, "Introduction to the issue on multitarget tracking," IEEE Journal of Selected Topics in Signal Processing, vol. 7, no. 3, pp. 373-375, 2013.

[2] Y. Bar-Shalom, F. Daum, and J. Huang, "The probabilistic data association filter: estimation in the presence of measurement origin uncertainty," IEEE Control Systems Magazine, vol. 29, no. 6, pp. 82-100, 2009.

[3] J. Liu, C. Han, F. Han, and Y. Hu, "Multiple maneuvering target tracking by improved particle filter based on multiscan JPDA," Mathematical Problems in Engineering, vol. 2012, Article ID 372161, 25 pages, 2012.

[4] S. S. Blackman, "Multiple hypothesis tracking for multiple target tracking," IEEE Aerospace and Electronic Systems Magazine, vol. 19, no. 1, pp. 5-18, 2004.

[5] T. Sathyan, T. J. Chin, S. Arulampalam, and D. Suter, "A multiple hypothesis tracker for multitarget tracking with multiple simultaneous measurements," IEEE Journal of Selected Topics in Signal Processing, vol. 7, no. 3, pp. 448-460, 2013.

[6] R. L. Popp, K. R. Pattipati, and Y. Bar-Shalom, "M-best S-D assignment algorithm with application to multitarget tracking," IEEE Transactions on Aerospace and Electronic Systems, vol. 37, no. 1, pp. 22-39, 2001.

[7] R. P. S. Mahler, "Multitarget bayes filtering via first-order multitarget moments," IEEE Transactions on Aerospace and Electronic Systems, vol. 39, no. 4, pp. 1152-1178, 2003.

[8] B.-N. Vo and W.-K. Ma, "The Gaussian mixture probability hypothesis density filter," IEEE Transactions on Signal Processing, vol. 54, no. 11, pp. 4091-4104, 2006.

[9] O. Cheng, H. Ji, and Y. Tian, "Improved Gaussian mixture CPHD tracker for multitarget tracking," IEEE Transactions on Aerospace and Electronic Systems, vol. 49, no. 2, pp. 1177-1191, 2013.

[10] O. Cappé, S. J. Godsill, and E. Moulines, "An overview of existing methods and recent advances in sequential Monte Carlo," Proceedings of the IEEE, vol. 95, no. 5, pp. 899-924, 2007.

[11] G. Casella and C. P. Robert, "Rao-Blackwellisation of sampling schemes," Biometrika, vol. 83, no. 1, pp. 81-94, 1996.

[12] S. Särkkä, A. Vehtari, and J. Lampinen, "Rao-Blackwellized Monte Carlo data association for multiple target tracking," in Proceedings of the 7th International Conference on Information Fusion (FUSION '04), pp. 583-590, Stockholm, Sweden, July 2004.

[13] S. Särkkä, A. Vehtari, and J. Lampinen, "Rao-Blackwellized particle filter for multiple target tracking," Information Fusion, vol. 8, no. 1, pp. 2-15, 2007.

[14] T. Schön, F. Gustafsson, and P.-J. Nordlund, "Marginalized particle filters for mixed linear/nonlinear state-space models," IEEE Transactions on Signal Processing, vol. 53, no. 7, pp. 22792289, 2005.

[15] S. Julier, J. Uhlmann, and H. F. Durrant-Whyte, "A new method for the nonlinear transformation of means and covariances in filters and estimators," IEEE Transactions Automatic Control, vol. 45 , no. 3 , pp. 477-482, 2000.

[16] W. Li and Y. Jia, "H-infinity filtering for a class of nonlinear discrete-time systems based on unscented transform," Signal Processing, vol. 90, no. 12, pp. 3301-3307, 2010.
[17] M. J. Grimble and A. Elsayed, "Solution of the $H_{\infty}$ optimal linear filtering problem for discrete-time systems," IEEE Transactions on Acoustics, Speech and Signal Processing, vol. 38, no. 7, pp. 1092-1104, 1990.

[18] I. Yaesh and U. Shaked, "A transfer function approach to the problems of discrete-time systems: $H_{\infty}$-optimal linear control and filtering," IEEE Transactions Automatic Control, vol. 36, no. 11, p. 1217, 1264-1271, 1991.

[19] B. Hassibi, A. H. Sayed, and T. Kailath, Indefinite-Quadratic Estimation and Control: A Unified Approach to $\mathrm{H}_{2}$ and $\mathrm{H}_{\infty}$ Theories, SIAM Press, 1999.

[20] J. Chandrasekar, A. J. Ridley, and D. S. Bernstein, "A comparison of the extended and unscented Kalman filters for discrete-time systems with nondifferentiable dynamics," in Proceedings of the American Control Conference, pp. 4431-4436, July 2007.

[21] F. Mustière, M. Bolić, and M. Bouchard, "Rao-Blackwellised particle filters: examples of applications," in Proceedings of the Canadian Conference on Electrical and Computer Engineering (CCECE '06), pp. 1196-1200, Ottawa, Canada, May 2006.

[22] A. Doucet, J. D. Freitas, and N. Gordon, Sequential Monte Carlo Methods in Practice, Springer, New York, NY, USA, 2001.

[23] A. Giremus, J.-Y. Tourneret, and V. Calmettes, "A particle filtering approach for joint detection/estimation of multipath effects on GPS measurements," IEEE Transactions on Signal Processing, vol. 55, no. 4, pp. 1275-1285, 2007.

[24] D. Simon, Optimal State Estimation: Kalman, $\mathrm{H}_{\infty}$ and Nonlinear Approaches, Wiley Press, 2006.

[25] U. Shaked and N. Berman, " $H_{\infty}$ nonlinear filtering of discretetime processes," IEEE Transactions on Signal Processing, vol. 43, no. 9, pp. 2205-2209, 1995.

[26] G. A. Einicke and L. B. White, "Robust extended Kalman filtering," IEEE Transactions on Signal Processing, vol. 47, no. 9, pp. 2596-2599, 1999.

[27] S. Julier, J. Uhlmann, and H. F. Durrant-Whyte, "A new method for the nonlinear transformation of means and covariances in filters and estimators," IEEE Transactions Automatic Control, vol. 45 , no. 3 , pp. $477-482,2000$.

[28] J. Chandrasekar, A. J. Ridley, and D. S. Bernstein, "A comparison of the extended and unscented Kalman filters for discrete-time systems with nondifferentiable dynamics," in Proceedings of the American Control Conference (ACC '07), pp. 4431-4436, July 2007.

[29] G. Sibley, G. Sukhatme, and L. Matthies, "The iterated sigma point Kalman filter with applications to long range stereo," in Proceedings of the 2nd Robotics: Science and Systems Conference, pp. 16-19, Philadelphia, Pa, USA, August 2006.

[30] A. Doucet, S. Godsill, and C. Andrieu, "On sequential Monte Carlo sampling methods for Bayesian filtering," Statistics and Computing, vol. 10, no. 3, pp. 197-208, 2000.

[31] G. Kitagawa, "Monte Carlo filter and smoother for nonGaussian nonlinear state space models," Journal of Computational and Graphical Statistics, vol. 5, no. 1, pp. 1-25, 1996.

[32] M. Bolić, P. M. Djurić, and S. Hong, "Resampling algorithms and architectures for distributed particle filters," IEEE Transactions on Signal Processing, vol. 53, no. 7, pp. 2442-2450, 2005. 




Advances in

Operations Research

mansans

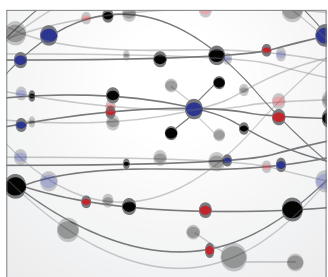

The Scientific World Journal
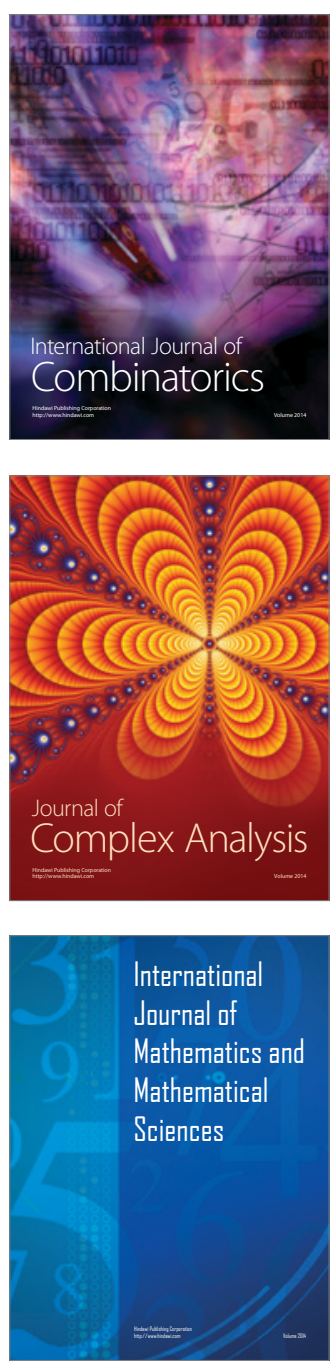
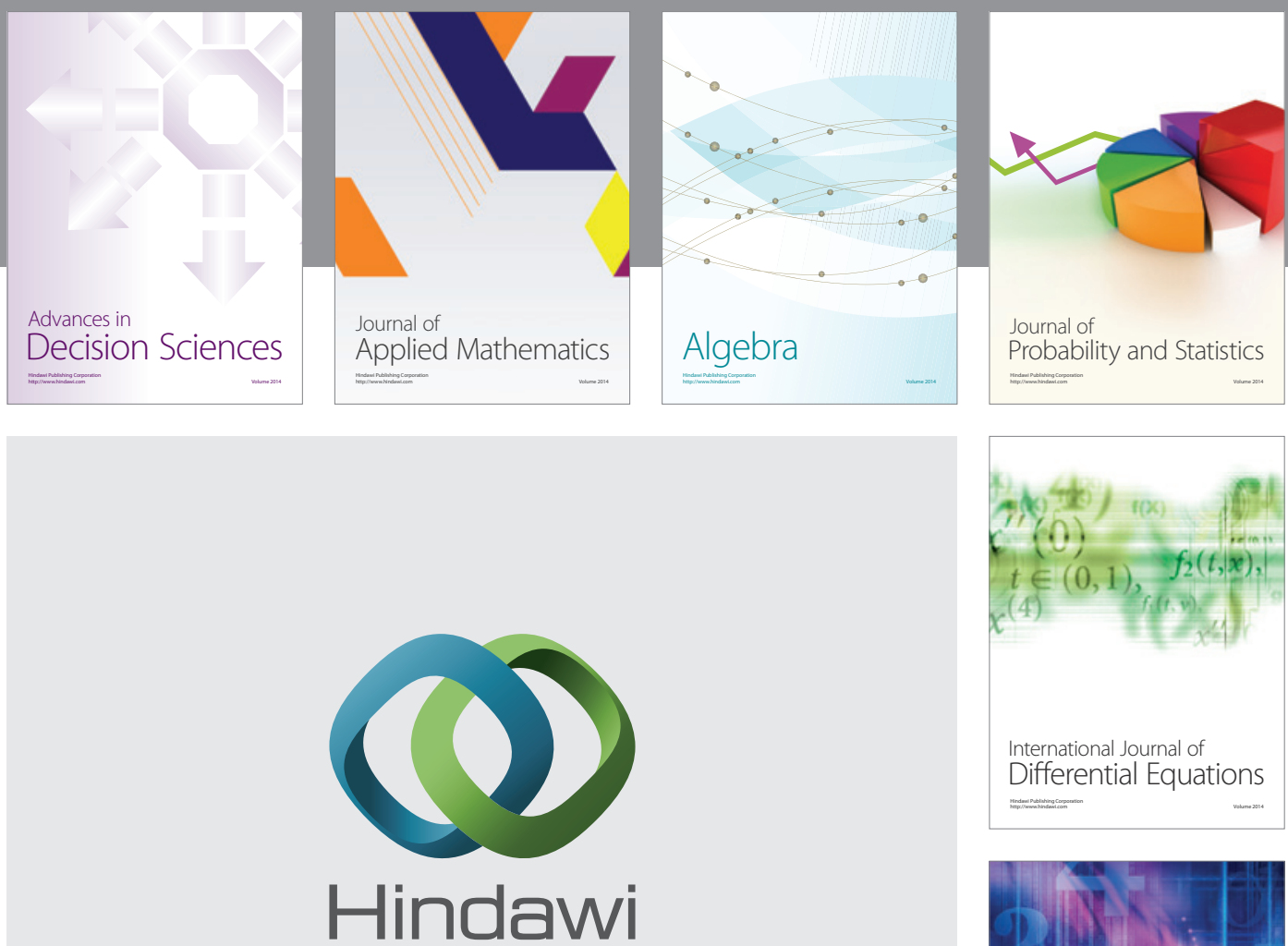

Submit your manuscripts at http://www.hindawi.com
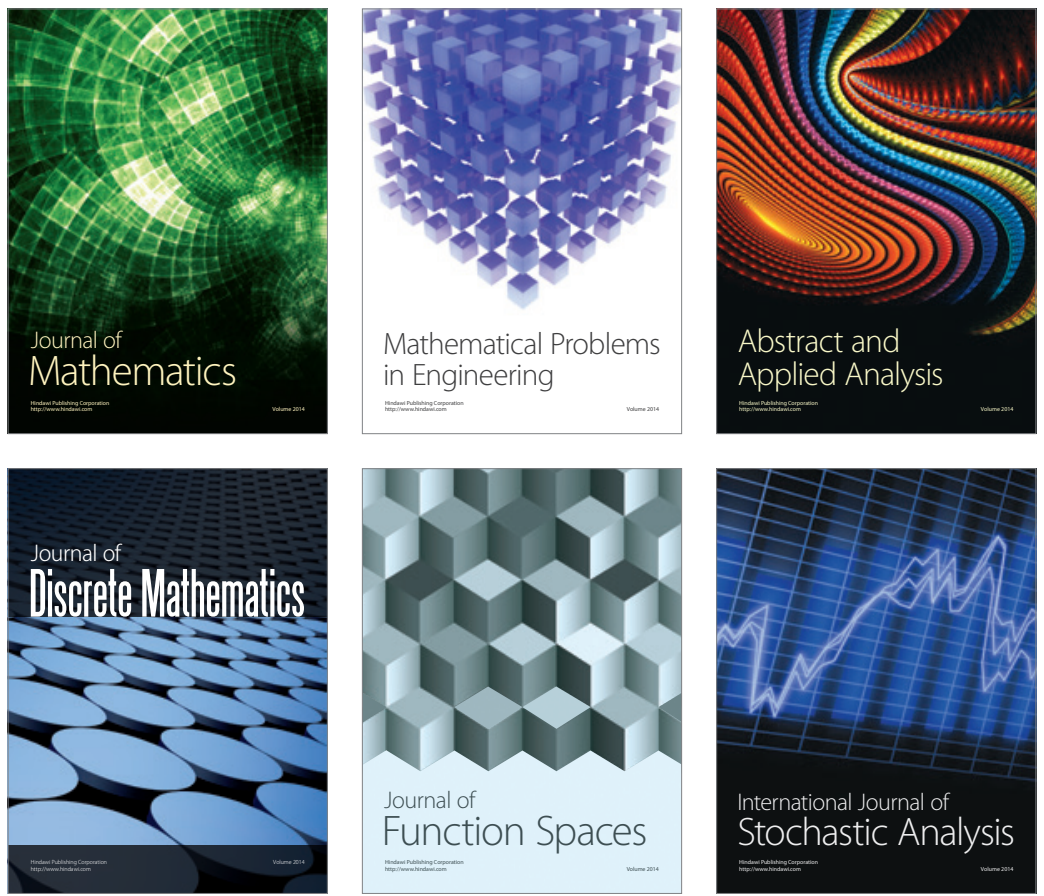

Journal of

Function Spaces

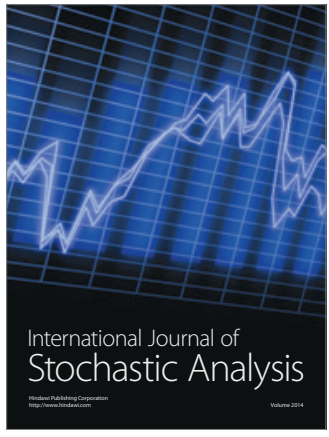

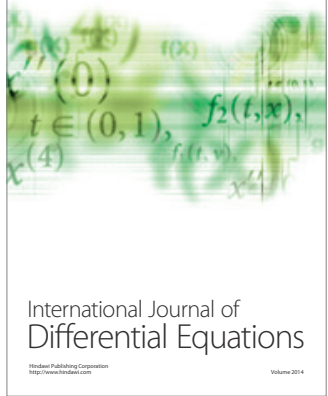
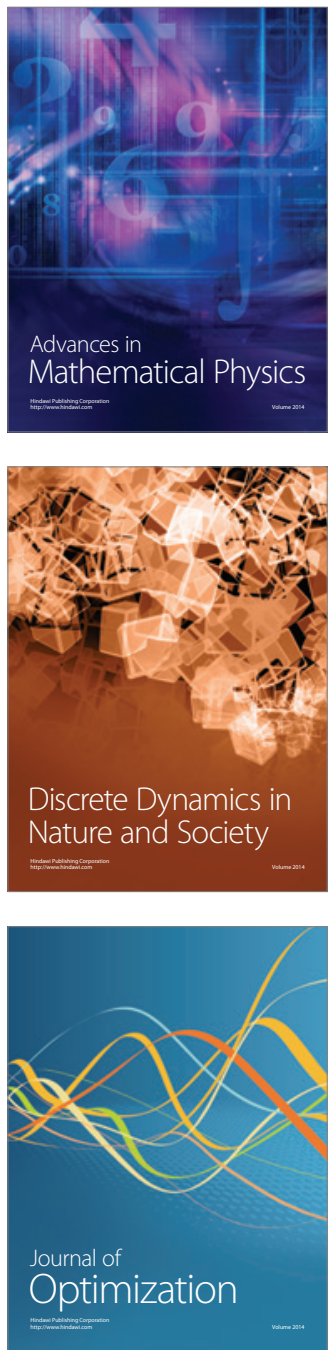